\title{
Bronchopulmonary Hemorrhage, CTCAE
}

National Cancer Institute

\section{Source}

National Cancer Institute. Bronchopulmonary Hemorrhage, CT CAE. NCI Thesaurus.

Code C143343.

A disorder characterized by bleeding from the bronchial wall and/or lung parenchyma. 